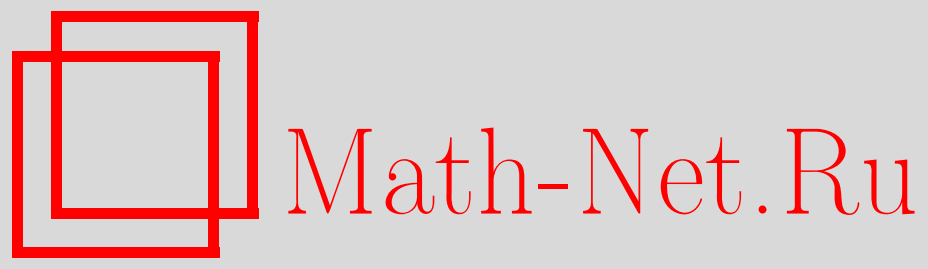

А. А. Кириллов, Семейные алгебры и обобщенные экспоненты для поливекторных представлений простых алгебр Ли типа $B_{n}$, Функи. анализ и его прил., 2008, том 42, выпуск 4, 72-82

DOI: https://doi.org/10.4213/faa2928

Использование Общероссийского математического портала Math$\mathrm{Net.Ru}$ подразумевает, что вы прочитали и согласны с пользовательским соглашением http://www . mathnet.ru/rus/agreement

Параметры загрузки:

IP : 52.6 .47 .48

26 апреля 2023 г., 05:12:51

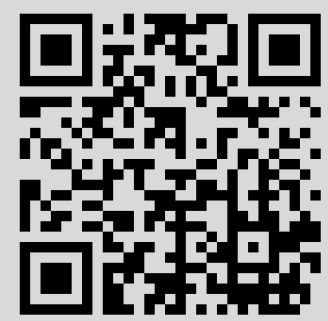


Функиионалъный анализ и его приложения

2008, т. 42, вып. 4, с. 72-82

УДК 512.815.1

\title{
Семейные алгебры и обобщенные экспоненты для поливекторных представлений простых алгебр Ли типа $B_{n}$
}

\author{
(c) 2008. А. А. Кириллов
}

К 95-летию моего дорогого учителя И. М. Гельфанда

\section{§1. Семейные алгебры}

1.1. Основные определения и факты. В [5], [6] автор ввел новый тип ассоциативных алгебр, связанных с простыми комплексными алгебрами Ли (или со связными приведенными системами корней). Здесь мы напомним основные свойства этих алгебр.

Пусть $\mathfrak{g}$ - простая комплексная алгебра Ли с обычным разложением

$$
\mathfrak{g}=\mathfrak{n}_{-} \oplus \mathfrak{h} \oplus \mathfrak{n}_{+} .
$$

Мы используем стандартные обозначения:

$P$ (соответственно $Q)$ - решетка весов (соответственно корневая решетка) в $\mathfrak{h}^{*}$;

$P_{+}$(соответственно $\left.Q_{+}\right)$- полугруппа, порожденная фундаментальными весами $\omega_{1}, \ldots, \omega_{l}$ (соответственно простыми корнями $\left.\alpha_{1}, \ldots, \alpha_{l}\right)$.

Для каждого веса $\lambda \in P_{+}$мы обозначаем через $\left(\pi_{\lambda}, V_{\lambda}\right)$ неприводимое представление алгебры Ли $\mathfrak{g}$ со старшим весом $\lambda$, а через $d(\lambda)$ - размерность пространства $V_{\lambda}$.

После выбора базиса в $V_{\lambda}$ пространство End $V_{\lambda}$ отождествляется с $\operatorname{Mat}_{d(\lambda)}(\mathbb{C})$ и представление $\pi_{\lambda}$ становится матричнозначной функцией на $\mathfrak{g}$. Пространство

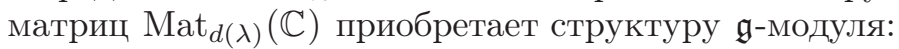

$$
X \cdot M=\left[\pi_{\lambda}(X), M\right], \quad X \in \mathfrak{g}, M \in \operatorname{Mat}_{d(\lambda)}(\mathbb{C}) .
$$

Напомним, что симметрическая алгебра $S(\mathfrak{g})$ и универсальная обертывающая алгебра $U(\mathfrak{g})$ тоже имеют (изоморфные) структуры $\mathfrak{g}$-модуля, которые продолжают присоединенное представление алгебры Ли $\mathfrak{g}$.

Пусть $G$ - связная и односвязная комплексная группа Ли с алгеброй Ли $\mathfrak{g}$. Она действует на всех $\mathfrak{g}$-модулях, описанных выше. Ассоциативные алгебры, введенные в [5], [6], - это два типа семейных алгебр:

Определение 1. Классическая семейная алгебра - это

$$
\mathscr{C}_{\lambda}(\mathfrak{g}):=\left(\operatorname{Mat}_{d(\lambda)}(\mathbb{C}) \otimes S(\mathfrak{g})\right)^{G},
$$

квантовая семейная алгебра - это

$$
\mathscr{Q}_{\lambda}(\mathfrak{g}):=\left(\operatorname{Mat}_{d(\lambda)}(\mathbb{C}) \otimes U(\mathfrak{g})\right)^{G} .
$$

Я надеюсь, что изучение этих алгебр окажется полезным в разных вопросах теории представлений полупростых алгебр Ли и их бесконечномерных аналогов. 
1.2. Геометрическая интерпретация. Первоначальная идея, приведшая к определению семейных алгебр, состояла в том, чтобы выделить из обертывающей алгебры $U(\mathfrak{g})$ одну или несколько изотипических компонент, которые бы сами образовывали алгебру (как, например, центр $Z(\mathfrak{g})$ алгебры $U(\mathfrak{g}))$. Нетрудно проверить, что в построении алгебр (2), (3) действительно участвует лишь конечное число изотипических компонент алгебр $S(\mathfrak{g})$ и $U(\mathfrak{g})$, а именно те, которые соответствуют детям данного представления $\pi_{\lambda}$ алгебры Ли $\mathfrak{g}$ (определение детей см. ниже).

Элемент $F \in \mathscr{C}_{\lambda}(\mathfrak{g})$ можно рассматривать как $G$-эквивариантное полиномиальное отображение из $\mathfrak{g}^{*}$ в $\operatorname{Mat}_{d(\lambda)}(\mathbb{C}) \simeq$ End $V_{\lambda}$. В самом деле, алгебра $S(\mathfrak{g})$ естественно изоморфна алгебре $\mathbb{C}\left[\mathfrak{g}^{*}\right]$ полиномиальных функций на $\mathfrak{g}^{*}$, так что $F$ - полиномиальная матричная функция на $\mathfrak{g}^{*}$. Из (2) вытекает, что эта функция эквивариантна, т. е.

$$
F(\operatorname{Ad} g X)=\pi(g) F(X) \pi(g)^{-1}, \quad g \in G, X \in \mathfrak{g} .
$$

Аналогичная интерпретация элементов квантовой семейной алгебры становится возможной, если мы будем рассматривать $U(\mathfrak{g})$ как алгебру полиномиальных функций на некотором квантовом многообразии $\widetilde{\mathfrak{g}}^{*}$ с некоммутативной алгеброй функций.

1.3. Элементы типа $\boldsymbol{M}$. Существует простой и красивый способ явного построения некоторых элементов семейных алгебр. Первый пример такого рода был давно известен специалистам по теории представлений и математической физике ${ }^{1)}$.

Пример 1. Пусть $\left\{X_{i}\right\}$ и $\left\{X^{i}\right\}$ - два дуальных базиса в алгебре Ли $\mathfrak{g}$ по отношению к какой-нибудь $(\operatorname{Ad} G)$-инвариантной невырожденной билинейной форме. Тогда матрица

$$
M=\pi\left(X^{i}\right) \otimes X_{i} \quad \text { (суммирование по индексу } i \text { подразумевается) }
$$

принадлежит $\mathscr{C}_{\lambda}(\mathfrak{g})$ (соответственно $\left.\mathscr{Q}_{\lambda}(\mathfrak{g})\right)$, если мы рассматриваем $X_{i}$ как элементы алгебры $S(\mathfrak{g})$ (соответственно $U(\mathfrak{g}))$.

Более общий факт был отмечен в [6]:

Лемма 1. Пусть $P$ есть $G$-инвариантный полином на $\mathfrak{g}^{*}$. Выберем ортонормированный базис $\left\{X_{i}\right\}$ в $\mathfrak{g}$ и будем рассматривать $X_{i}$ как координаты на $\mathfrak{g}^{*}$. Пусть $\partial^{i}$ означает частную производную $\partial / \partial X_{i}$. Тогда отображение

$$
M_{P}(X)=\pi\left(X^{i}\right) \otimes\left(\partial_{i} P\right)(X)
$$

$G$-эквивариантно. Более того, оно является элементом центра алгебры $\mathscr{C}_{\lambda}(\mathfrak{g})$.

Предыдущий пример является частным случаем, когда $P=\frac{1}{2}\left(X_{i}\right)^{2}$.

Эта удобная конструкция не переносится буквально на квантовые семейные алгебры, но взамен можно предложить другую конструкцию. Воспользуемся тем, что $U(\mathfrak{g})$ - алгебра Хопфа, т. е. обладает гомоморфизмом коумножения

$$
\Delta: U(\mathfrak{g}) \rightarrow U(\mathfrak{g}) \otimes U(\mathfrak{g}), \quad \mathfrak{g} \ni X \mapsto X \otimes 1+1 \otimes X .
$$

Используя $\Delta$, мы можем определить гомоморфизм

$$
\varphi=(\pi \otimes \mathrm{Id}) \circ \Delta: U(\mathfrak{g}) \rightarrow \operatorname{Mat}_{d(\lambda)}(\mathbb{C}) \otimes U(\mathfrak{g}) .
$$

1)Этот пример был одной из мотиваций введения семейных алгебр. 
Поскольку это отображение, очевидно, $G$-эквивариантно, мы получаем следующий результат.

Теорема 1. Образ иентра $Z(\mathfrak{g})$ алгебры $U(\mathfrak{g})$ относителъно $\varphi$ содержится в квантовой семейной алгебре $\mathscr{Q}_{\lambda}(\mathfrak{g})$.

Пример 2. Для элемента $A=\frac{1}{2} X^{i} X_{i} \in Z(\mathfrak{g})$ мы получаем $\varphi(A)=\pi(A) \otimes 1+$ $M_{A}+1 \otimes A$.

Элементы, полученные описанными двумя конструкциями, мы называем элементами типа $M$. Они принадлежат центру семейной алгебры и в «хороших» случаях порождают всю эту алгебру.

1.4. Дети неприводимого представления алгебры Ли g. Рассмотрим подробнее $\mathfrak{g}$-модуль $V_{\lambda} \otimes V_{\lambda}^{*} \simeq \operatorname{End} V_{\lambda} \simeq \operatorname{Mat}_{d(\lambda)}(\mathbb{C})$. Разложим пространство $\operatorname{Mat}_{d(\lambda)}(\mathbb{C})$ на неприводимые относительно действия $\mathfrak{g}$ подпространства $W_{1}, \ldots, W_{r}$. Обозначим старшие веса этих компонент через $\mu_{1}, \ldots, \mu_{r}$. Заметим, что вес $\mu$ и дуальный вес $\mu^{*}$ входят в этот список с одинаковыми кратностями, поскольку представление $\pi_{\lambda} \otimes \pi_{\lambda}^{*}$ самодвойственно. В дальнейшем нам будет удобно считать, что в пространстве $W_{k}$ действует представление $\pi_{\mu_{k}^{*}}$

Мы называем представления $\pi_{\mu_{i}}$ детъми представления $\pi_{\lambda}$. Если некоторый старший вес встречается несколько раз, мы говорим, что имеются близнецы.

Заметим также, что если $\lambda=\lambda^{*}$, то пространство $\operatorname{Mat}_{d(\lambda)}(\mathbb{C})$ можно разложить $\mathfrak{g}$-инвариантным образом в сумму двух подпространств. А именно, если $\pi_{\lambda}$ ортогонально, то инвариантные подпространства суть подпространства симметрических и кососимметрических матриц. Если же $\pi_{\lambda}$ симплектично относительно кососимметрической формы с матрицей $J$, то инвариантные подпространства суть подпространства $J$-симметрических и $J$-кососимметрических матриц. Поэтому множество детей можно разделить на две части: сыновья заполняют пространство $(J$-)симметрических матриц, а дочери - пространство $(J$-)кососимметрических матриц. Из самодвойственности каждой части вытекает, что каждому ребенку $\mu$ соответствует двойственный ребенок $\mu^{*}$ того же пола (который может и совпадать с $\mu$ ).

1.5. Представления с простым спектром. Предположим, что все веса представления $\pi_{\lambda}$ простые. Тогда образ картановской подалгебры $\mathfrak{h} \subset \mathfrak{g}$ в $\operatorname{Mat}_{d(\lambda)}(\mathbb{C})$ содержит матрицу $D$ с различными собственными значениями. Выбирая весовой базис в $V_{\lambda}$, мы можем считать эту матрицу диагональной. Поэтому для каждого отображения $F \in \mathscr{C}_{\lambda}(\mathfrak{g})$ значения его ограничения на $\mathfrak{h}$ перестановочны с $D$ и, следовательно, диагональны. Мы видим, что коммутатор двух элементов классической семейной алгебры обращается в нуль на (любой) картановской подалгебре $\mathfrak{h}$. Тогда он равен нулю на всех полупростых элементах из $\mathfrak{g}$ и, значит, везде. Таким образом (см. [6], где получен более общий результат), справедлива

Теорема 2. Если представление $\left(\pi_{\lambda}, V_{\lambda}\right)$ имеет простой спектр, то алгебра $\mathscr{C}_{\lambda}(\mathfrak{g})$ коммутативна и порождается элементом (5).

Для квантовых алгебр $\mathscr{Q}_{\lambda}(\mathfrak{g})$ это также верно, поскольку они являются полупростыми деформациями классических алгебр. 


\section{§2. Семейные алгебры и обобщенные экспоненты}

Строение $S(\mathfrak{g})$ как $\mathfrak{g}$-модуля описывается известной теоремой Б. Костанта:

$$
S(\mathfrak{g}) \simeq I(\mathfrak{g}) \otimes H(\mathfrak{g}),
$$

где $I(\mathfrak{g}) \simeq \mathbb{C}\left[\mathfrak{g}^{*}\right]^{G}$ - алгебра $G$-инвариантных полиномиальных функций на $\mathfrak{g}^{*} \simeq \mathfrak{g}$, а $H(\mathfrak{g})$ - пространство гармонических полиномиальных функций на $\mathfrak{g}^{*}$, отождествляемое с алгеброй полиномиальных функций $\mathbb{C}[N]$ на нильпотентном конусе $N \subset \mathfrak{g}$. Таким образом, изотипическая компонента $S_{\mu}(\mathfrak{g})$ имеет вид $I(\mathfrak{g}) \otimes$ $H_{\mu}(\mathfrak{g})$, т. е. является свободным модулем над $I(\mathfrak{g})$.

Пространство $H_{\mu}(\mathfrak{g})$ конечномерно, и кратность представления $\pi_{\mu}$ в нем равна кратности нулевого веса в представлении $\left(\pi_{\mu}, V_{\mu}\right)$.

Поскольку $H_{\mu}(\mathfrak{g})$ наследует из $\mathbb{C}[N]$ градуировку степенями полиномов, $H_{\mu}(\mathfrak{g})=\bigoplus_{s \geqslant 0} H_{\mu}^{s}(\mathfrak{g})$, мы можем рассматривать кратность $E_{\mu}$ представления $\pi_{\mu}$ в $H_{\mu}(\mathfrak{g})$ не как число, а как полином от $q$ с целыми неотрицательными коэффициентами,

$$
E_{\mu}:=\sum_{k=1}^{m} q^{e_{k}(\mu)}=\sum_{i} s_{i} q^{i} .
$$

Числа $e_{1}(\mu) \geqslant \cdots \geqslant e_{m}(\mu)$ называются обобщенными экспонентами представления $\pi_{\mu}$. Ясно, что двойственные представления имеют один и тот же набор экспонент.

Пространство $H_{\mu}^{i}(\mathfrak{g})$ разлагается (неоднозначно) в сумму $s_{i}$ неприводимых подпространств $H_{\mu, k}$, для которых $e_{k}=i$, так что $H_{\mu}=\bigoplus_{k=1}^{m} H_{\mu, k}$.

$\mathrm{K}$ сожалению, вычисление кратности веса и его $q$-аналога остается одной из вечных задач теории представлений, для решения которой есть красивая формула, но нет простого общего алгоритма.

В случае присоединенного представления $(\mathrm{Ad}, \mathfrak{g})=\left(\pi_{\theta}, V_{\theta}\right)$ обобщенные экспоненты совпадают с обычными экспонентами группы Вейля (или системы корней). Их число равно рангу алгебры Ли $\mathfrak{g}$, и есть несколько простых алгоритмов их вычисления.

Пользуясь введенными понятиями, мы можем дать явное описание классической семейной алгебры $\mathscr{C}_{\pi_{\lambda}}(\mathfrak{g})$ в терминах детей $\pi_{\lambda}$ и их обобщенных экспонент. Для этого заметим, что пространство

$$
\left(W_{k}^{*} \otimes H_{\mu, p}^{i}\right)^{G} \simeq \operatorname{Hom}_{G}\left(W_{k}, H_{\mu, p}^{i}\right) \simeq \operatorname{Hom}_{G}\left(W_{k}, V_{\mu}\right)
$$

одномерно по лемме Шура. Выберем в нем базисный вектор $\alpha_{i, \mu, p}$.

В результате мы получаем

$$
\mathscr{C}_{\lambda}(\mathfrak{g})=I(\mathfrak{g}) \otimes\left(\sum_{k} W_{k} \otimes \sum_{i, \mu, p} H_{\mu, p}^{i}\right)^{G}=\bigoplus_{i, \mu, p} I(\mathfrak{g}) \alpha_{i, \mu, p} .
$$

Если, кроме того, известно, что $\pi_{\lambda}$ - представление с простым спектром (как это имеет место для спинорных представлений ниже), мы получаем следующий результат:

Теорема 3. Семейная алгебра $\mathscr{C}_{\lambda}(\mathfrak{g})$ для представления $\pi_{\lambda}$ с простьм спектром является коммутативной алгеброй и свободным модулем ранга $d(\lambda)$ над $I(\mathfrak{g})$. 


\section{§3. Представления алгебры Ли $\mathrm{B}_{n}$}

3.1. Алгебра нечетных дифференциальных операторов. Пусть $\xi^{1}, \ldots, \xi^{n}-$ нечетные переменные, удовлетворяющие антикоммутационным соотношениям

$$
\xi^{i} \xi^{j}+\xi^{j} \xi^{i}=0 \text { для всех } i, j .
$$

Обозначим через $\operatorname{Gras}_{n}:=\mathbb{C}\left\{\xi^{1}, \ldots, \xi^{n}\right\}$ комплексную ассоциативную алгебру с единицей, порожденную этими переменными. Ясно, что $\operatorname{dim} \operatorname{Gras}_{n}=2^{n}$. Мы обозначим через $\partial_{i}, 1 \leqslant i \leqslant n$, нечетные дифференцирования алгебры $\operatorname{Gras}_{n}$, действующие на образующие $\xi^{j}$ по формуле

$$
\partial_{i} \xi^{j}=\delta_{i}^{j}
$$

Ассоциативную алгебру операторов на пространстве $\operatorname{Gras}_{n}$, порожденную умножениями на $\xi^{j}$ и дифференцированиями $\partial_{i}$, обозначим через $W_{n}$ и назовем ее элементы нечетными дифференциальными операторами.

Лемма 2. Алгебра $W_{n}$ изоморфна полной матричной алгебре $\operatorname{Mat}_{2^{n}}(\mathbb{C})$.

Доказательство. Мы покажем, что алгебра $W_{n}$ действует на пространстве $\operatorname{Gras}_{n}$ неприводимо и, следовательно, совпадает с алгеброй всех линейных операторов. Для этого достаточно показать, что для любых двух элементов $P, Q$ из $\operatorname{Gras}_{n}$ найдется оператор $A \in W_{n}$, такой, что $A P=Q$. В самом деле, пусть $P=\sum_{I} c_{I} \xi^{I}$, где $I$ пробегает все упорядоченные по возрастанию подмножества в $\{1, \ldots, n\}, \xi^{I}=\xi^{i_{1}} \cdots \xi^{i_{k}}$, а $c_{I}$ - произвольные комплексные коэффициенты. Выберем одно из $J$ максимальной мощности среди тех, для которых $c_{J} \neq 0$. Тогда оператор $\partial_{J}=\partial_{j_{1}} \cdots \partial_{j_{m}}$ переводит $P$ в ненулевую константу $c_{J}$. Теперь достаточно положить $A=c_{J}^{-1} Q \partial_{J}$.

Как и всякая ассоциативная алгебра, $W_{n}$ является алгеброй Ли относительно операции коммутирования. В этом ее качестве мы обозначаем ее через $\operatorname{Lie}\left(W_{n}\right)$. Обозначим через $L_{n}$ подалгебру Ли в $\operatorname{Lie}\left(W_{n}\right)$, порожденную умножениями на $\xi^{j}$ и дифференцированиями $\partial_{i}$. Нетрудно проверить, что следующие $2 n^{2}+n$ элементов образуют базис в $L_{n}$ :

$$
\xi^{i}, \quad \partial_{j}, \quad a_{j}^{i}:=\frac{1}{2}\left[\xi^{i}, \partial_{j}\right], \quad b^{i, j}:=\frac{1}{2}\left[\xi^{i}, \xi^{j}\right], \quad c_{i, j}:=\frac{1}{2}\left[\partial_{i}, \partial_{j}\right],
$$

где $i, j$ пробегают значения от 1 до $n$, а в двух последних выражениях подразумевается, что $i<j$.

Лемма 3. Алгебра Ли $L_{n}$ изоморфна $\mathbf{B}_{n} \simeq s o(2 n+1, \mathbb{C})$.

Доказательство. Напомним, что алгебра Ли $\mathfrak{g}=s o(2 n+1, \mathbb{C})$ определяется как совокупность всех антисимметричных комплексных матриц $X$ порядка $2 n+1$. Технически удобно рассматривать матрицы, антисимметричные относительно второй диагонали, образующие алгебру Ли, изоморфную g. Пусть $e_{i, j}$, $-n \leqslant i, j \leqslant n,-$ стандартные матричные единицы; тогда матрицы $X_{i, j}:=$ $e_{i, j}-e_{-j,-i}$ лежат в $\mathfrak{g}$ и удовлетворяют соотношениям

$$
X_{i, j}=-X_{-j,-i} \text {. }
$$

Матрицы $X_{i, j}, i+j>0$, образуют базис в $\mathfrak{g}$.

Искомый изоморфизм между $L_{n}$ и $\mathfrak{g}$ имеет вид

$$
\frac{1}{\sqrt{2}} \xi^{i} \leftrightarrow X_{i, 0}, \quad \frac{1}{\sqrt{2}} \partial_{j} \leftrightarrow X_{0, j}, \quad a_{j}^{i} \leftrightarrow X_{i, j}, \quad b^{i, j} \leftrightarrow X_{i,-j}, \quad c_{i, j} \leftrightarrow X_{-i, j},
$$


где $1 \leqslant i, j \leqslant n$.

$G$-инвариантное скалярное произведение в $\mathbf{B}_{n}$ мы нормализуем так:

$$
(A, B)=-\frac{1}{2} \operatorname{tr}(A B) \text {. }
$$

Мы выберем в качестве картановской подалгебры $\mathfrak{h} \subset \mathfrak{g}$ подалгебру диагональных матриц. Базис в ней составляют матрицы

$$
H_{k}:=X_{k, k}=e_{k, k}-e_{-k,-k}, \quad 1 \leqslant k \leqslant n,
$$

которые при изоморфизме с $L_{n}$ переходят в операторы

$$
h_{k}:=a_{k}^{k}=\frac{1}{2}\left[\xi^{k}, \partial_{k}\right]=\xi^{k} \partial_{k}-\frac{1}{2} .
$$

Отметим, что $\left\{H_{1}, \ldots, H_{n}\right\}$ образуют ортонормированный базис в $\mathfrak{h}$.

Весами определяющего представления $\pi_{1}$ алгебры Ли $\mathfrak{g}$ являются 0 и $\pm H_{k}$, $1 \leqslant k \leqslant n$.

Корни алгебры Ли $\mathfrak{g}$, т. е. ненулевые веса представления $\pi_{2}$, имеют вид $\pm H_{k}$ и $\pm H_{i} \pm H_{j}, i<j$.

Хорошо известно, что все фундаментальные представления алгебры Ли $\mathfrak{g}$, за исключением одного, имеют вид $\pi_{k}=\wedge^{k} \pi_{1}, 1 \leqslant k<n$, и старший вес $\omega_{k}=\sum_{i=1}^{k} H_{i}$. Представление $\pi_{n}=\wedge^{n} \pi_{1}$ не является фундаментальным, и его старший вес $\sum_{i=1}^{n} H_{i}$ вдвое больше, чем последний фундаментальный вес $\omega_{n}=\frac{1}{2} \sum_{i=1}^{n} H_{i}$ (см. ниже).

3.2. Спинорное представление $\boldsymbol{\sigma}$ алгебры Ли $\mathfrak{g}$. Построенный выше изоморфизм алгебр Ли $\mathfrak{g}$ и $L_{n}$ позволяет определить представление $\sigma$ алгебры Ли $\mathfrak{g}$ в пространстве $\operatorname{Gras}_{n}$, которое называется спинорным представлением. Оно неприводимо, поскольку образом алгебры $U(\mathfrak{g})$ служит вся алгебра $W_{n}=$ End $V_{n}$.

Лемма 4. Веса спинорного представления имеют вид

$$
\frac{ \pm H_{1} \pm \cdots \pm H_{n}}{2} \quad \text { любым выбором знаков. }
$$

Доказательство. Воспользуемся соотношением $h_{j} \xi^{i}=\left(\delta_{j}^{i}-\frac{1}{2}\right) \xi^{i}$. По правилу Лейбница (точнее, по его нечетному аналогу)

$$
h_{j} \xi^{I}= \begin{cases}\frac{1}{2} \xi^{I}, & \text { если } j \in I, \\ -\frac{1}{2} \xi^{I}, & \text { если } j \notin I,\end{cases}
$$

откуда и вытекает требуемая формула.

Мы видим также, что старший вес представления $\sigma$ совпадает с $\omega_{n}$ и поэтому $\sigma$ является последним фундаментальным представлением алгебры Ли $\mathfrak{g}$.

Заметим, что все $2^{n}$ весов представления $\sigma$ различны, так что теорема 3 применима к семейной алгебре $\mathscr{C}_{\omega_{n}}(\mathfrak{g})$, которая, следовательно, является свободной коммутативной алгеброй над $I(\mathfrak{g})$ ранга $2^{n}$.

3.3. Дети спинорного представления алгебры Ли $\mathbf{B}_{\boldsymbol{n}}$. Мы начнем с замечания, что спинорное представление $\sigma$ самодвойственно, поскольку на пространстве $\operatorname{Gras}_{n}$ существует g-инвариантное скалярное произведение. Его 
можно задать с помощью так называемого интеграла Березина от полинома от антикоммутирующих переменных. По определению интеграл Березина от полинома $P=\sum_{I} c_{I} \xi^{I}$ равен старшему коэффициенту $c_{\{1, \ldots, n\}}$ :

$$
\int_{\mathrm{Ber}} P=c_{\{1, \ldots, n\}} \text {. }
$$

Определим в $\operatorname{Gras}_{n}$ антиинволюцию $P \mapsto \widehat{P}$, которая переводит $\xi^{i}$ в $-\xi^{i}$ и соответственно $\xi^{i_{1}} \xi^{i_{2}} \cdots \xi^{i_{k}}$ в $(-1)^{k} \xi^{i_{k}} \xi^{i_{k-1}} \cdots \xi^{i_{1}}$. Искомое скалярное произведение имеет вид

$$
(P, Q)=\int_{\mathrm{Ber}} \widehat{P} Q=(-1)^{\frac{k(k+1)}{2}} \int_{\mathrm{Ber}} P Q, \quad \text { где } k=\operatorname{deg} P .
$$

Оно обладает следующим свойством:

$$
(P, Q)=(-1)^{\frac{n(n+1)}{2}}(Q, P),
$$

так что спинорное представление ортогонально при $n=0,3 \bmod 4$ и симплектично при $n=1,2 \bmod 4$.

Веса представления $\sigma \otimes \sigma^{*} \simeq \sigma \otimes \sigma$ имеют вид

$$
\sum_{k=1}^{n} \varepsilon_{k} H_{k} \quad \text { где } \varepsilon_{k}=1,0,-1 \text {. }
$$

Среди них доминантными являются только $\theta_{k}=\sum_{i=1}^{k} H_{i}, 0 \leqslant k \leqslant n$. Кратность веса $\theta_{k}$ равна $2^{n-k}$, поскольку именно столькими способами можно записать $\theta_{k}$ в виде суммы (или разности) двух весов представления $\sigma$ (см. (16)).

Лемма 5. Представление $\pi_{k}$ со старшим весом $\theta_{k}$ содержит вес $\theta_{l}$ только при $l \leqslant k$, и соответствующая кратность равна $\left(\begin{array}{c}n-l \\ m\end{array}\right)$, где $m=\left[\frac{k-l}{2}\right]$.

Доказательство. В самом деле, искомая кратность равна числу способов записать вектор $\theta_{l}$ как сумму $k$ различных весов представления $\pi_{1}$. Если $k-l=$ $2 m$, то такая запись имеет вид

$$
\theta_{l}=H_{1}+\cdots+H_{l}+\sum_{s=1}^{m}\left(H_{i_{s}}+\left(-H_{i_{s}}\right)\right) .
$$

Если же $k-l=2 m+1$, то она выглядит так:

$$
\theta_{l}=H_{1}+\cdots+H_{l}+0+\sum_{s=1}^{m}\left(H_{i_{s}}+\left(-H_{i_{s}}\right)\right) .
$$

В обоих случаях множество $\left\{i_{1}, \ldots, i_{m}\right\}$ пробегает все $m$-точечные подмножества в $\{l+1, \ldots, n\}$. Поэтому искомая кратность равна $\left(\begin{array}{c}n-l \\ m\end{array}\right)$.

Утверждение леммы и еще некоторые простые факты относительно кратностей весов $\theta_{k}$ в разных представлениях удобно записать в виде таблицы (см. табл. 1). 


\begin{tabular}{|c|c|c|c|c|c|c|c|}
\hline & $\theta_{n}$ & $\theta_{n-1}$ & $\theta_{n-2}$ & $\theta_{n-3}$ & $\theta_{n-s}$ & $\theta_{1}$ & $\theta_{0}$ \\
\hline$S^{2}(\sigma)$ & 1 & 1 & 2 & 4 & $2^{s-1}$ & $2^{n-2}$ & $2^{n-1}$ \\
\hline$\wedge^{2}(\sigma)$ & 0 & 1 & 2 & 4 & $2^{s-1}$ & $2^{n-2}$ & $2^{n-1}$ \\
\hline$\pi_{n}$ & 1 & 1 & 1 & 1 & 1 & 1 & 1 \\
\hline$\pi_{n-1}$ & 0 & 1 & 2 & 3 & $s$ & $n-1$ & $n$ \\
\hline$\pi_{n-2}$ & 0 & 0 & 1 & 3 & $\left(\begin{array}{c}s \\
2\end{array}\right)$ & $\left(\begin{array}{c}n-1 \\
2\end{array}\right)$ & $\left(\begin{array}{c}n \\
2\end{array}\right)$ \\
\hline$\pi_{n-3}$ & 0 & 0 & 0 & 1 & $\left(\begin{array}{c}s \\
3\end{array}\right)$ & $\left(\begin{array}{c}n-1 \\
3\end{array}\right)$ & $\left(\begin{array}{c}n \\
3\end{array}\right)$ \\
\hline \hline$\pi_{n-t}$ & 0 & 0 & 0 & 0 & $\left(\begin{array}{c}s \\
t\end{array}\right)$ & $\left(\begin{array}{c}n-1 \\
t\end{array}\right)$ & $\left(\begin{array}{c}n \\
t\end{array}\right)$ \\
\hline \hline$\pi_{1}$ & 0 & 0 & 0 & 0 & 0 & 1 & $n$ \\
\hline$\pi_{0}$ & 0 & 0 & 0 & 0 & 0 & 0 & 1 \\
\hline
\end{tabular}

Табл. 1

Анализируя эту таблицу, мы приходим к следующему выводу:

Теорема 4. Разложения представлений $\sigma \otimes \sigma, S^{2} \sigma$ и $\wedge^{2} \sigma$ на неприводимъе компоненты имеют следующий вид:

(a) $\sigma \otimes \sigma=\bigoplus_{k=0}^{n} \pi_{k}$

(b) $S^{2}(\sigma)=\pi_{n} \oplus \pi_{n-3} \oplus \pi_{n-4} \oplus \pi_{n-7} \oplus \cdots=\sum_{s \equiv 0,3 \bmod 4} \pi_{n-s}$;

(c) $\wedge^{2}(\sigma)=\pi_{n-1} \oplus \pi_{n-2} \oplus \pi_{n-5} \oplus \pi_{n-6} \oplus \cdots=\sum_{s \equiv 1,2 \bmod 4} \pi_{n-s}$.

3.4. Обобщенные экспоненты детей спинорного представления. Из леммы 5 мы уже знаем число обобщенных экспонент для всех представлений $\pi_{k}, 0 \leqslant k \leqslant n$, т. е. для всех детей спинорного представления. А именно, $\pi_{k}$ имеет $\left(\begin{array}{c}n \\ m\end{array}\right)$ экспонент, где $m=\left[\frac{k}{2}\right]$. Наша задача теперь - найти правильный $q$-аналог этого выражения. Напрашивающийся ответ - гауссов биномиальный коэффициент $\left[\begin{array}{c}n \\ m\end{array}\right]_{q}$ - оказывается в этом случае неверным! Правильный ответ дается следующей неожиданной (по крайней мере, для меня) формулой.

Теорема 5. Производящая функиия для обобщенных экспонент представления $\pi_{k}$ со стариим весом $\theta_{k}$ в зависимости от четности $k$ есть

$$
E_{\theta_{2 m}}(q)=q^{m}\left[\begin{array}{c}
n \\
m
\end{array}\right]_{q^{2}} \quad \text { или } \quad E_{\theta_{2 m+1}}(q)=q^{n-m}\left[\begin{array}{l}
n \\
m
\end{array}\right]_{q^{2}} .
$$

Доказательство теоремы будет приведено ниже, и при этом мы получим также полезную информацию о соответствующей классической семейной алгебре $\mathscr{C}_{\omega_{n}}\left(\mathbf{B}_{\mathbf{n}}\right)$.

\section{§4. Ограничение на картановскую подалгебру}

Для доказательства основной теоремы мы рассмотрим подробнее ограничение элемента $F \in \mathscr{C}_{\omega_{n}}(\mathfrak{g})$ на $\mathfrak{h}^{*}$. Это ограничение является полиномиальной функцией от координат $H_{1}, \ldots, H_{n}$, значения которой - диагональные матрицы порядка $2^{n}$. Элементы этих матриц, как и базис в $\mathrm{Gras}_{n}$, удобно нумеровать последовательностями $\varepsilon=\left\{\varepsilon_{1}, \ldots, \varepsilon_{n}\right\}, \varepsilon_{k}= \pm 1$. 
Пусть $f$ - полином, стоящий в первой клетке, где все $\varepsilon_{k}$ равны 1 . Условие ковариантности (4) показывает, что полином, стоящий в клетке $\varepsilon$, равен $f\left(\varepsilon_{1} H_{1}, \ldots, \varepsilon_{n} H_{n}\right)$. Таким образом, элемент $F$ полностью определяется полиномом $f$. Мы получаем таким образом вложение $\alpha$ классической семейной алгебры $\mathscr{C}_{\omega_{n}}(\mathfrak{g})$ в алгебру полиномов от $H_{1}, \ldots, H_{n}$.

Лемма 6. Образ вложения $\alpha$ совпадает с алгеброй $\mathscr{A}_{n}$ всех симметрических полиномов от $H_{1}, \ldots, H_{n}$.

В самом деле, из условия ковариантности следует, что полином $f=\alpha(F)$ инвариантен относительно группы $S_{n}$ перестановок переменных. Остается показать, что любой симметрический полином получается из какого-нибудь элемента семейной алгебры.

Сначала проверим утверждение леммы для подалгебры $\mathscr{B}_{n} \subset \mathscr{A}_{n}$, состоящей из симметрических полиномов от $H_{1}^{2}, \ldots, H_{n}^{2}$. Алгебра $\mathscr{B}_{n}$ является алгеброй инвариантов для группы Вейля алгебры Ли g. Напомним, что эта группа порождается перестановками переменных и изменениями их знаков. Образующими алгебры $\mathscr{B}_{n}$ являются ограничения на $\mathfrak{h}^{*}$ полиномов

$$
\Delta_{k}:=\operatorname{tr} M^{2 k}
$$

Легко видеть, что скалярные матрицы вида $\Delta_{k} \cdot 1$ принадлежат семейной алгебре и $\alpha\left(\Delta_{k} \cdot 1\right)=\left.\Delta_{k}\right|_{\mathfrak{h}^{*}}$.

Далее, алгебра $\mathscr{A}_{n}$ как градуированный модуль над $\mathscr{B}_{n}$ имеет полином Пуaнкаре

$$
P(q)=\frac{\prod_{k=1}^{n}\left(1-q^{2 k}\right)}{\prod_{k=1}^{n}\left(1-q^{k}\right)}=\prod_{k=1}^{n}\left(1+q^{k}\right) .
$$

В частности, ранг этого модуля равен $2^{n}$.

С другой стороны, ранг семейной алгебры над $\mathscr{B}_{n}$ равен общему числу экспонент всех детей спинорного представления, которое также равно $2^{n}$ как размерность пространства векторов нулевого веса в $\operatorname{End}\left(\operatorname{Gras}_{n}\right)$. Лемма доказана.

Вернемся теперь к разбиению пространства $\operatorname{Mat}_{2^{n}}(\mathbb{C}) \simeq \operatorname{End}\left(\operatorname{Gras}_{n}\right)$ на неприводимые относительно $\sigma(G)$ подпространства $W_{k}, 0 \leqslant k \leqslant n$, в которых действуют представления $\pi_{k}$. Каждое $W_{k}$ имеет нетривиальное пересечение с пространством $D$ диагональных матриц. Более точно, размерность этого пересечения равна числу экспонент представления $\pi_{k}$. Каждой такой экспоненте $e$ соответствует элемент $F_{e, k}$ степени $e$ семейной алгебры, связанный с ребенком $\pi_{k}$. Симметрические полиномы $f_{e, k}=\alpha\left(F_{e, k}\right)$ образуют однородный базис в алгебре $\mathscr{A}_{n}$ как $\mathscr{B}_{n}$-модуле.

Окончательно вопрос о вычислении экспонент сводится к нахождению однородного базиса $\mathscr{B}_{n}$-модуля $\mathscr{A}_{n}$. Как известно, одним из базисов $\mathscr{A}_{n}$ как комплексного векторного пространства является набор мономиальных симметрических функций $m_{a}=m_{a_{1} \cdots a_{n}}, a_{i} \in \mathbb{Z}_{+}$. По определению $m_{a}$ является суммой всех различных мономов вида $H_{1}^{a_{1}} \cdots H_{n}^{a_{n}}$. Назовем типом такой функции число $t\left(m_{a}\right)$ нечетных индексов среди $\left\{a_{1}, \ldots, a_{n}\right\}$. Линейную оболочку всех мономиальных функций типа $t$ обозначим через $\mathscr{A}_{n}^{(t)}$. Ясно, что $\mathscr{A}_{n}=\bigoplus_{t=0}^{n} \mathscr{A}_{n}^{(t)}$. 
Теорема 6. (а) Пространство $\mathscr{A}_{n}^{(t)}$ - свободный градуированнъй модуль над градуированной алгеброй $\mathscr{B}_{n}$. Многочлен Пуанкаре этого модуля имеет вид

$$
P_{t}(q)=q^{t}\left[\begin{array}{l}
n \\
t
\end{array}\right]_{q^{2}} .
$$

(b) Наименьшая экспонента представления $\pi_{k}$ равна

$$
s(k)= \begin{cases}\frac{k}{2}, & \text { если } k \text { четно, } \\ n-\frac{k-1}{2}, & \text { если } k \text { нечетно. }\end{cases}
$$

(с) Пусть $m$ - мономиальная функиия типа $t$. Тогда совокупность вектоpoв

$$
\vec{m}:=\left\{m\left(\varepsilon_{1} H_{1}, \ldots, \varepsilon_{n} H_{n}\right)\right\}
$$

для всевозможных значений $H_{1}, \ldots, H_{n}$ заполняет пространство $W_{k} \cap D$, где $k$ определяется из равенства $s(k)=t(m)$.

(d) Производящая функиия для обобщенных экспонент представления $\pi_{k}$ совпадает с градуированным рангом модуля $\mathscr{A}_{n}^{(s(k))}$.

Схема доказательства. Первое утверждение теоремы вместе с вычислением полинома Пуанкаре получается с помощью прямого вычисления ряда Пуанкаре градуированного пространства $\mathscr{A}_{n}^{(t)}$.

Рассмотрим утверждение (c). Для всех мономиальных функций типа $t$ векторы (23) заполняют одно и то же подпространство $D_{t}$ в $D$, поскольку система линейных уравнений, определяющая это подпространство, зависит только от $t$. Таким образом, мы получаем два разбиения пространства $D$ в прямую сумму подпространств,

$$
D=\bigoplus_{t=0}^{n} D_{t} \quad \text { и } \quad D=\bigoplus_{k=0}^{n} D \cap W_{k} .
$$

Легко понять, что каждый элемент второго разбиения целиком содержится в одном из элементов первого разбиения. Но число элементов обоих разбиений одинаково, поэтому они совпадают. Осталось вычислить зависимость между двумя параметрами: $t=s(k)$. Смысл параметра $t$ ясен из того, что это - минимальная степень мономиальной функции данного типа. Таким образом, $s(k)$ - это минимальная экспонента представления $\pi_{k}$. Зная число экспонент представления $\pi_{k}$ и полиномы Пуанкаре $\mathscr{B}_{n}$-модулей $\mathscr{A}_{n}^{(t)}$, мы определяем $t=s(k)$ с точностью до замены на $n-t$. Окончательную ясность вносит рассмотрение максимальной экспоненты представления $\pi_{k}$, которая без труда вычисляется по формуле Костанта [7, формула 5.3.5] и равна высоте старшего веса $\sum_{i=1}^{k} H_{i}$, т. е. $\frac{k(2 n+1-k)}{2}$.

Базис в семейной алгебре нумеруется подмножествами в $\{1, \ldots, n\}$. Подмножеству $\left\{i_{1}<\cdots<i_{k}\right\}$ соответствует элемент, у которого в левом верхнем углу стоит мономиальная функция $m_{a}, a=\left\{a_{1} \geqslant \cdots \geqslant a_{n} \geqslant 0\right\}$, минимального веса, у которой индексы $a_{i_{1}}, \ldots, a_{i_{k}}$ (и только они) нечетны. Всего таких функций $\left(\begin{array}{l}n \\ k\end{array}\right)$, а производящий полином для их степеней равен $q^{k}\left[\begin{array}{l}n \\ k\end{array}\right]^{2}$. 


\section{§5. Скалярное произведение Рани Брылински}

В заключение рассмотрим еще один подход к описанию экспонент. В этом контексте он был предложен Рани Брылински в [3]. Идея состоит в том, что число экспонент $E_{\pi}$ или его $q$-аналог $E_{\pi}(q)$ является в какой-то мере показателем величины представления. Поскольку он аддитивен по $\pi$, величину

$$
E\left(\pi_{1}, \pi_{2}\right):=E_{\pi \otimes \pi_{2}^{*}}(q)
$$

можно рассматривать как своеобразное скалярное произведение в кольце представлений, обладающее полезным свойством

$$
E\left(\pi_{1} \otimes \pi, \pi_{2}\right)=E\left(\pi_{1}, \pi^{*} \otimes \pi_{2}\right) .
$$

В работе [2] показано, что в случае простых групп Ли типа $\mathbf{A}_{n}$ это скалярное произведение выражается явно в терминах полиномов Макдональда.

В общем случае свойства этого скалярного произведения кодируются производящей функцией

$$
\sum_{\pi \in \widehat{G}} \chi_{\pi}(t) E_{\pi}(q)
$$

на максимальном торе $T \subset G$ (или его компактной форме). Если положить $q=1$, искомая функция легко вычисляется с помощью интегральной формулы Г. Вейля. Она равна $\frac{|W|}{\Delta(t)}$, где $|W|-$ порядок группы Вейля, а $\Delta-$ знаменатель в формуле Вейля для характера. Вполне вероятно, что справедлив $q$-аналог этой формулы.

\section{ЛитературА}

[1] Е. Б. Дынкин, Некоторые свойства систем весов линейных представлений полупростых групп Ли, Докл. АН СССР, 71:2 (1950), 221-224.

[2] R. K. Brylinski (Gupta), Limits of weight spaces, Lusztig's q-analogs, and fibering of adjoint orbits, J. Amer. Math. Soc., 2:3 (1989), 517-533.

[3] R. K. Gupta, Generalized exponents via Hall-Littlewood symmetric functions, Bull. Amer. Math. Soc., 16:2 (1987), 287-291.

[4] W. Hesselink, Characters of nullcone, Math. Ann., 252:3 (1980), 179-182.

[5] A. A. Kirillov, Family algebras, Electron. Res. Announc. Amer. Math. Soc., 6:1 (2000), 7-20 (electronic).

[6] A. A. Kirillov, Introduction to family algebras, Moscow Math. J., 1:1 (2001), 49-63.

[7] B. Kostant, Lie group representations on polynomial rings, Amer. J. Math., 85 (1963), 327-404.

[8] G. Lusztig, Singularities, character formulas, and a q-analogue of Kostant's weight multiplicity formula, in: Analyse et Topologie sur les Espaces Singuliers (II-III), Asterisque, vol. 101-102, Soc. Math. France, Paris, 1983, 208-227.

[9] D. Panyushev, Weight multiplicity free representations, $\mathfrak{g}$-endomorphism algebras and Dynkin polynomials, J. London Math. Soc., 69:2 (2004), 273-290.

Институт проблем передачи информации РАН Department of Mathematics, The University of Pennsylvania e-mail: kirillov@math.upenn.edu
Поступило в редакцию 26 июня 2006 г. 